\title{
LncRNA PCGEM1 mediates oxaliplatin resistance in hepatocellular carcinoma via miR-129-5p/ETV1 axis in vitro
}

\author{
Jie Chen ${ }^{1,2, A, D, F}$, Daiyue Yuan ${ }^{2, A-C}$, Qingya Ha0 ${ }^{2, C, D}$, Dongmei Zhu $^{2, C}$, Zhong Chen ${ }^{1,3, E}$ \\ ${ }^{1}$ Department Of General Surgery, The First Affiliated Hospital of Soochow University, Suzhou, China \\ 2 Department Of General Surgery, The Second Affiliated Hospital of Nantong University, China \\ ${ }^{3}$ Department of General Surgery, Affiliated Hospital of Nantong University, China \\ A - research concept and design; B - collection and/or assembly of data; C - data analysis and interpretation; \\ $D$ - writing the article; $E$ - critical revision of the article; $F$ - final approval of the article
}

\section{Address for correspondence}

Zhong Chen

E-mail:chenz9806@163.com

Funding sources

Youth Science Project of Nantong City (grant No. WKZL2018014).

\section{Conflict of interest}

None declared

Received on September 28, 2020

Reviewed on February 21, 2021

Accepted on April 7, 2021

Published online on July 20, 2021

\begin{abstract}
Background. Hepatocellular carcinoma (HCC) is one of the most severe malignant cancers that leads to high death rate worldwide. Recent research revealed that long non-coding RNAs (IncRNAs) exert a critical role regarding chemoresistance in numerous cancers, including HCC.

Objectives. Our research aimed to explore the function and molecular mechanism of IncRNA PCGEM7 on oxaliplatin resistance of HCC in vitro.

Materials and methods. Expression of the IncRNA PCGEM1, together with miR-129-5p, and the mRNA level of ETV1 and drug resistance-related genes including LRPA, MDRT and MDR3 were determined using quantitative real-time polymerase chain reaction (qRT-PCR) in an oxaliplatin-resistant HCC cell line (Hep3B/ OXA). Cell Counting Kit-8 (CCK-8) was employed to assess the viability and cell survival rate, and transwell assays were performed to measure the number of migrated or invaded cells. In addition, the relation among IncRNA PCGEM1, miR-129-5p and ETV1 were determined using luciferase assay.
\end{abstract}

Results. Our data indicated that PCGEM1 and ETV1 expression were enhanced in Hep3B/OXA cells. Furthermore, knockdown of IncRNA PCGEM7 significantly decreased the migration, invasion and mRNA expressions of $\angle R P A, M D R 1$ and MDR3, and the cell viability in Hep3B/OXA cells. The starBase online tool and luciferase assays verified that miR-129-5p targeted PCGEM1 and ETV1, signifying that PCGEM1 could enhance ETV1 expression via suppressing miR-129-5p.

Conclusions. Our findings demonstrated that PCGEM1 modulated oxaliplatin resistance by targeting the miR129-5p/ETV7 pathway in HCC in vitro, suggesting a potential strategy for the treatment of chemoresistant HCC.

Key words: hepatocellular carcinoma, miR-129-5p, oxaliplatin resistance, PCGEM1, ETV1

Cite as

Chen J, Yuan D, Hao Q, Zhu D, Chen Z. LncRNA PCGEMI

mediates oxaliplatin resistance in hepatocellular carcinoma

via miR-129-5p/ETV1 axis in vitro. Adv Clin Exp Med.

2021;30(8):831-838. doi:10.17219/acem/135533

DOI

10.17219/acem/135533

\section{Copyright}

Copyright by Author(s)

This is an article distributed under the terms of the

Creative Commons Attribution 3.0 Unported (CC BY 3.0)

(https://creativecommons.org/licenses/by/3.0/) 


\section{Background}

Hepatocellular carcinoma $(\mathrm{HCC})$ is a severe type of malignant tumor, leading to a large number of cancer-related deaths worldwide. ${ }^{1,2}$ It is an invasive and fast-growing tumor that leads to higher recurrence and metastasis. ${ }^{3}$ Despite advancements in the therapeutic techniques for $\mathrm{HCC}$ such as radiotherapy, chemotherapy and surgery, the prognosis is still unsatisfactory, with an overall five-year survival rate of $15-40 \% .{ }^{4,5}$ The underlying mechanism behind the pathogenesis of HCC is complicated and needs to be investigated for promising prognosis and early-stage detection. ${ }^{6}$ Therefore, novel targets and diagnostic indicators of $\mathrm{HCC}$ treatment are urgently needed.

Along with the progression of microarray analysis and sequencing, numerous novel non-coding RNAs have been discovered. Non-coding RNAs are sequences of nucleotides transcribed from chromatin and lack protein-coding capacity. ${ }^{7}$ Long non-coding RNAs (lncRNAs) are a group of non-coding RNAs having a length of $>200$ nucleotides. ${ }^{8}$ Previous research showed that lncRNAs exert critical functions in the pathogenesis of $\mathrm{HCC} .{ }^{9}$ For example, lncRNA UBE2CP3 was overexpressed in HCC and enhanced tumor metastasis through the modulation of EMT and induced migration and invasion. ${ }^{10}$ Furthermore, other research revealed that overexpression of lncRNA UCA1 suppressed miR-216b expression and stimulated FGFR1/ ERK pathways to enhance the pathogenesis of $\mathrm{HCC}{ }^{11}$ Moreover, lncRNA AK021443 mediated the migration and proliferation of HCC cells via EMT regulation in vitro. ${ }^{12}$ The lncRNA PCGEM1 was identified to be highly expressed in prostate cancer, and acts to enhance its proliferation. ${ }^{13,14}$ Also, PCGEM1 was highly expressed in ovarian cancer tissues and enhanced the invasion, migration and proliferation, while decreasing the apoptosis rate. ${ }^{15}$ Furthermore, PCGEM1 induced metastasis and EMT via enhancing the SNAI1 expression in gastric cancer cells. ${ }^{16}$ However, whether lncRNA PCGEM1 exerts its function in the development and tumorigenesis of $\mathrm{HCC}$ remains unknown.

It has been previously reported that oxaliplatin-mediated chemotherapy prolonged survival and reduced mortality during treatment of HCC patients in an advanced stage of the disease. ${ }^{17}$ Conversely, the resistance mechanism of oxaliplatin still needs to be elucidated. Numerous research has revealed that lncRNAs play a vital role in chemotherapy resistance in HCC. Several lncRNAs were highly expressed in chemoresistant HCC tissues and cells, and were shown to exert their function during oxaliplatin resistance and HCC pathogenesis. ${ }^{18}$ For example, lncRNA ARSR increased doxorubicin resistance in HCC by regulating PTEN.$^{2}$ Conversely, IncRNA HULC increased chemosensitivity by inhibiting autophagy in $\mathrm{HCC}$ cells. ${ }^{19}$

\section{Objectives}

This study aimed to investigate the impact of lncRNA PCGEM1 on oxaliplatin resistance in vitro in an HCC cell line. Our findings elucidated that lncRNA PCGEM1 mediates oxaliplatin resistance in HCC via the miR-129-5p/ ETV1 axis, indicating that PCGEM1 might be a therapeutic target for HCC treatment.

\section{Materials and methods}

\section{Cell culture}

The human liver cancer Hep3B cell line was acquired from American Type Cell Culture (ATCC, Manassas, USA) and cultured in Dulbecco's modified Eagle's medium (DMEM; Biocompare, San Francisco, USA) containing 100 units $/ \mathrm{mL}$ penicillin, $100 \mu \mathrm{g} / \mathrm{mL}$ streptomycin (Thermo Fisher Scientific, Waltham, USA) and 10\% fetal bovine serum (FBS; Biocompare) at $37^{\circ} \mathrm{C}$ in $5 \% \mathrm{CO}_{2}$.

\section{Establishment of oxaliplatin-resistant Hep3B cells}

Hep3B cells were exposed to increasing concentrations of oxaliplatin $(2-25 \mu \mathrm{M})$ to establish the oxaliplatin-resistant Hep3B cells. ${ }^{20}$ The $\mathrm{IC}_{50}$ of Hep3B cells was calculated after detection with Cell Counting Kit-8 (CCK-8) (Beyotime, Shanghai, China), and after that, the oxaliplatin $(25 \mu \mathrm{M})$ group was selected as Hep3B/OXA. Hep3B and Hep3B/OXA cells were cultured in minimum essential medium (MEM) containing 10\% FBS. A T25 culture flask was used to seed cells $\left(2 \times 10^{6}\right.$ cells/well $)$ for $72 \mathrm{~h}$ per passage.

\section{Cell transfection}

Briefly, scrambled siRNA (si-control) and PCGEM1 siRNAs (si-PCGEM1\#1), (si-PCGEM1\#2) and (si-PCGEM1\#3), and pcDNA3.1 control (pcDNA/control) were all obtained from Invitrogen (Carlsbad, USA). The $m i R-129-5 p$ mimics, mimic negative control (NC), $m i R-129-5 p$ inhibitor, and inhibitor NC was bought from GeneCopoeia (Rockville, USA). ETV1 mRNA 3'UTR and NC were obtained from Origene (Rockville, USA). Hep3B cells were cultured with $60-70 \%$ confluence at $37^{\circ} \mathrm{C}$ in $5 \% \mathrm{CO}_{2}$, and Lipofectamine 2000 (Invitrogen) was used to transfect these plasmids into the cells following the manufacturer's instructions (http:// go.microsoft.com/fwlink/p/?LinkId=255141).

\section{Mycoplasma detection}

Hep3B cells and Hep3B/OXA cells were kept in an RPMI1640 medium containing 10\% FBS and antibiotic-antimycotic solution (Spectrum Chemical Manufacturing Corp., 
New Brunswick, USA) and cultured for 3 years with occasional freezing as previously described. ${ }^{21}$ Blood agar plates and Mycoplasma IST2 kit (Biomereux Italia Spa, Florence, Italy) were used to perform titer of mycoplasmas as described previously. ${ }^{21}$ Twelve well plates were used to add mycoplasmas $\left(1 \times 10^{5} \mathrm{CFU} / \mathrm{mL}\right)$ into the mycoplasma-free Hep3B cells $\left(2 \times 10^{3}\right.$ cells/well $)$. Infected cells were analyzed using MycoGuard ${ }^{\mathrm{TM}}$ Mycoplasma PCR detection kit (GeneCopoeia) for the detection of mycoplasmas using the polymerase chain reaction (PCR) method, as described previously. ${ }^{21}$ Agarose gel electrophoresis was used to visualize amplified PCR products.

\section{CCK-8 assay}

Chemosensitivity and the rate of cell survival were examined using a CCK-8 assay. More specifically, 24-well plates were used to seed the cells at a density of $2 \times 10^{3}$ cells/well at $37^{\circ} \mathrm{C}$. The CCK-8 solution $(10 \mu \mathrm{L}$; Abcam, Cambridge, USA) was introduced into wells, and these plates were incubated for $1 \mathrm{~h}$ at $37^{\circ} \mathrm{C}$. Proliferation was determined at $460 \mathrm{~nm}$. The cell growth inhibition curve was detected by calculating the value of $\mathrm{IC}_{50}$ as (1-OD/ $\left.\mathrm{OD}_{0 \mu \mathrm{M}}\right) \times 100 \%$, as described previously. ${ }^{22}$

\section{Transwell invasion and migration assay}

Transwell chambers (Becton Dickinson, Franklin Lakes, USA) were used to perform invasion and migration assay on Hep3B/OXA cells. For performing the invasion assay, Hep3B/OXA cells were passaged in a serum-free media $(200 \mu \mathrm{L})$ and kept in the higher compartment, followed by $600 \mu \mathrm{L}$ of a medium comprising $10 \%$ FBS into the lower compartment. The cells were fixed with polyoxymethylene after incubation for $48 \mathrm{~h}$. For the migration assay, 24-well plates were used to seed Hep3B/OXA cells, and $1 \times 10^{6}$ cells were re-suspended in a serum-free media $(200 \mu \mathrm{L})$ and kept in the higher compartment. Media $(600 \mu \mathrm{L})$ with $10 \%$ FBS were introduced into the lower compartment, and cells were incubated with $5 \% \mathrm{CO}_{2}$ for $48 \mathrm{~h}$ at $37^{\circ} \mathrm{C}$. Finally, the invasion and migration of cells was visualized after staining with $20 \%$ Giemsa solution (Sigma-Aldrich, St. Louis, USA) and counted from 5 random chambers using an inverted microscope (Nikon Corp., Tokyo, Japan). All the experimentations were repeated in triplicate.

\section{Luciferase assay}

Online bioinformatics tools including miRcode (http:// mircode.org/), starBase v. 2.0 (http://starbase.sysu.edu. cn/starbase2/index.php) and microRNA.org (https://bigd. big.ac.cn/databasecommons/database/id/1426) were used to predict the binding sites of $m i R-129-5 p$ on PCGEM1 as well as the ETV1 binding sites on the $m i R-129-5 p$ gene. Luciferase assay (BioAssay Systems, Hayward, USA) was then performed to assess the luciferase activity according to the manufacturer's protocol (https://www.bioassaysys. com/Luciferase-Reporter-Assay-Kit-(1000-tests).html). A Mammalian Genomic DNA Miniprep Kit (Qiagen, Hilden, Germany) was used to generate the pGL3 plasmid (VWR, Radnor, USA), and once bound to their binding sites on the $m i R-129-5 p$, the RNA sequence of PCGEM1 and 3'-UTR of ETV1 were spliced into pGL3 to produce pGL3-PCGEM1 (or ETV1 mRNA) mutant or wild-type reporter vector. The 24-well plates were used to seed cells, and pGL3 mutant or wild type vector (100 ng) and miR-129-5p mimic or mimic NC, miR-129-5p inhibitor or inhibitor negative control $(50 \mathrm{nmol} / \mathrm{L})$ were transfected using Lipofectamine 2000 (Thermo Fisher Scientific) into the cells. Renilla luciferase gene was taken as a standard. All the experiments were performed in triplicate.

\section{qRT-PCR}

Total RNA was extracted using Ribozol RNA extraction reagent (VWR) following the manufacturer's protocol (https://us.vwr.com/store/product/7437721/vwr-lifescience-ribozoltm-rna-extraction-reagent). A NanoDrop ND-1000 spectrophotometer (Thermo Fisher Scientific) was used to examine the purity and RNA concentration at 260/280 nm. Reverse transcription of total RNA $(2 \mu \mathrm{g})$ into cDNA was performed using the SuperScript First-Stand Synthesis System. ABI PRISM 7500 and SYBR Premix ExTaq II kit (Thermo Fisher Scientific) was used to perform quantitative real-time PCR (qRT-PCR). Primers sequences are given below in Table 1 . All the experiments were performed 3 times. GAPDH acted as the internal control, and the expressions of genes were measured using the $2^{-\Delta \Delta C t}$ method..$^{23}$

Table 1. The primer sequences for quantitative real-time polymerase chain reaction ( $q R T-P C R)$ analysis

\begin{tabular}{|c|c|c|}
\hline \multicolumn{2}{|c|}{ Primers } & Sequence \\
\hline \multirow{2}{*}{ PCGEM 1} & forward & CTGTGTCTGCAACTTCCTCTAA \\
\hline & reverse & TCCCAGTGCATCTCGTAGTA \\
\hline \multirow{2}{*}{ miR-129-5p } & forward & ACCCAGTGCGATTTGTCA \\
\hline & reverse & ACTGTACTGGAAGATGGACC \\
\hline \multirow{2}{*}{ ETV1 } & forward & TAGCCGTTCACTCCGCTATT \\
\hline & reverse & CCTCGTTGATGTGACGTTCC \\
\hline \multirow{2}{*}{ LRP1 } & forward & AATGGGCTAAGCCTGGACAT \\
\hline & reverse & TGCCACTCCGATACTCAGTC \\
\hline \multirow{2}{*}{ MDR1 } & forward & TCATTCGAGTAGCGGCTCTT \\
\hline & reverse & TTCTTTGCTCCTCCATTGCG \\
\hline \multirow{2}{*}{ MDR3 } & forward & TGCAGCCCACTTATTCATGC \\
\hline & reverse & TTCTTCACCTCCAGGCTCAG \\
\hline \multirow{2}{*}{ GAPDH } & forward & GGTGTGAACCATGAGAAGTATGA \\
\hline & reverse & GAGTCCTTCCACGATACCAAAG \\
\hline
\end{tabular}




\section{Statistical analyses}

Data are presented as the mean \pm standard deviation (SD). IBM SPSS v. 19 (IBM Corp., Armonk, USA) and GraphPad Prism v. 8.0 (GraphPad Software, San Diego, USA) were used for statistical analyses of all data. The difference between the 2 groups was evaluated using Student's t-test while multiple-group comparisons were performed using analysis of variance (ANOVA) and Tukey's post hoc analysis. The general alpha value was set as 0.05 . All the experimentations were performed 3 times.

\section{Results}

\section{Effect of IncRNA PCGEM1 and ETV1 on oxaliplatin-resistant HCC cells}

Hep3B cells were treated with oxaliplatin, and the $P C$ GEM1 expression was examined using qRT-PCR. Results indicated that PCGEM1 expression was significantly enhanced in Hep3B/OXA cells compared to Hep3B cells without oxaliplatin treatment (Fig. 1A). The survival of Hep3B cells was assessed using a CCK-8 assay, and our data indicated that the percentage survival of Hep3B/ OXA cells was greater than Hep3B control cells (Fig. 1B). Furthermore, ETV1 mRNA expression was markedly enhanced in Hep3B/OXA cells compared to that of parental Hep3B cells (Fig. 1C). Next, oligonucleotides were
A

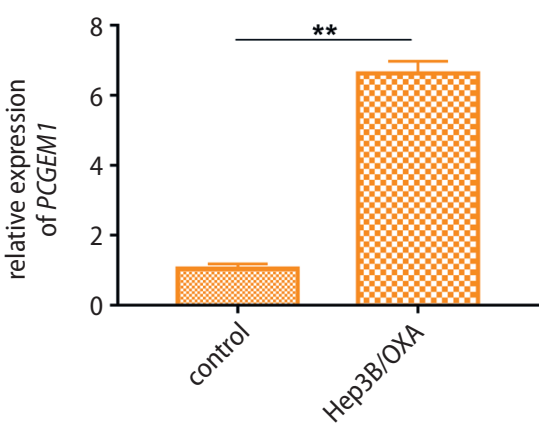

B

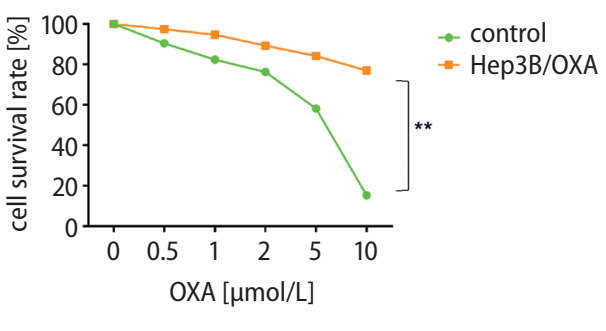

C

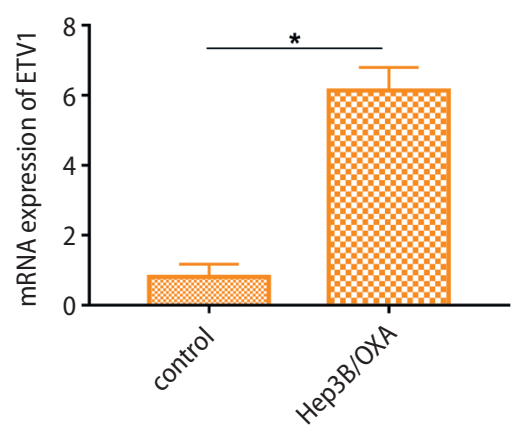

D
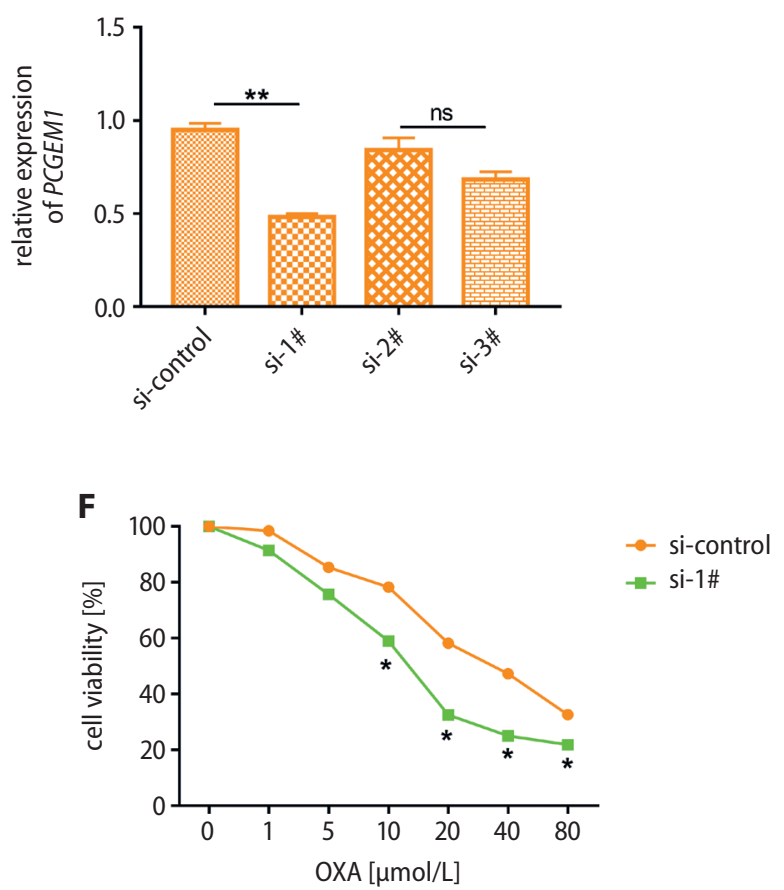

E
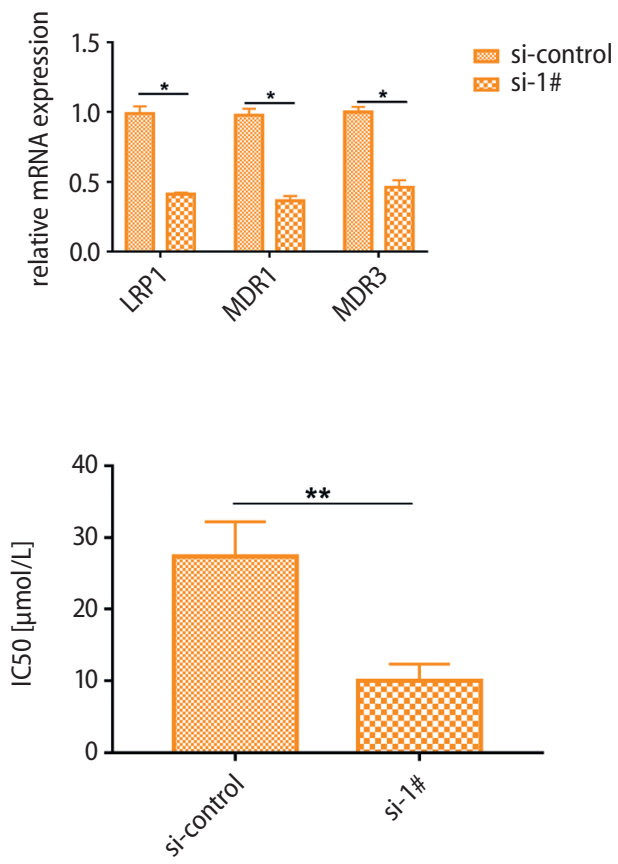

Fig. 1. Effect of IncRNA PCGEM1 and ETV1 in oxaliplatin-resistant hepatocellular carcinoma (HCC) cells. A. PCGEM1 expression was determined in oxaliplatinresistant Hep3B cells using quantitative real-time polymerase chain reaction (qRT-PCR); B. The cell survival rate was examined in oxaliplatin-resistant Hep3B cells using the Cell Counting Kit-8 (CCK-8) assay; C. The ETV1 mRNA level in oxaliplatin-resistant Hep3B cells was examined using qRT-PCR; D. The PCGEM1 expression was assessed after Hep3B cells were treated with interfering oligonucleotides (** $p<0.01$, ns - not significant); E. qRT-PCR measured the mRNA level of MDRT, MDR3 and LRP1 (drug resistance-related genes); F. The IC $\mathrm{C}_{50}$ was measured in oxaliplatin-resistant Hep3B cells $\left({ }^{*} \mathrm{p}<0.03,{ }^{* *} \mathrm{p}<0.002\right)$ 
A

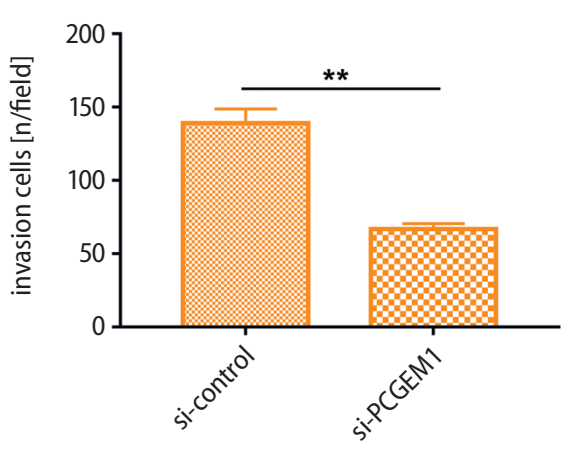

B

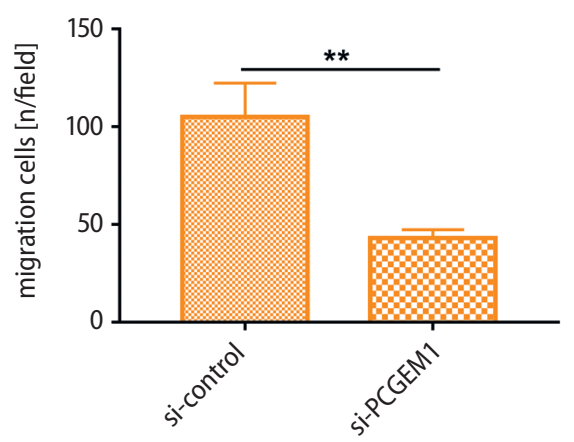

Fig. 2. Effect of PCGEM1 knockdown on invasion and migration of oxaliplatinresistant hepatocellular carcinoma $(\mathrm{HCC})$ cells in vitro. A. Transwell assay detected the number of invasive cell in Hep3B/ OXA cells transfected with PCGEM1 siRNA compared to the cells transfected with empty vector (** $p<0.002)$; B. Transwell assay detected the number of migrative cell in Hep3B/OXA cells transfected with PCGEM1 siRNA compared to the cells transfected with empty vector ${ }^{* *} p<0.002$
A

Target: 5 ' cuacuCCUAGGGCAGCAAAAAg 3'

$|1:||:||||||| \mid$

miRNA : 3' cguucGGGU-CUGGCGUUUUUC 5'
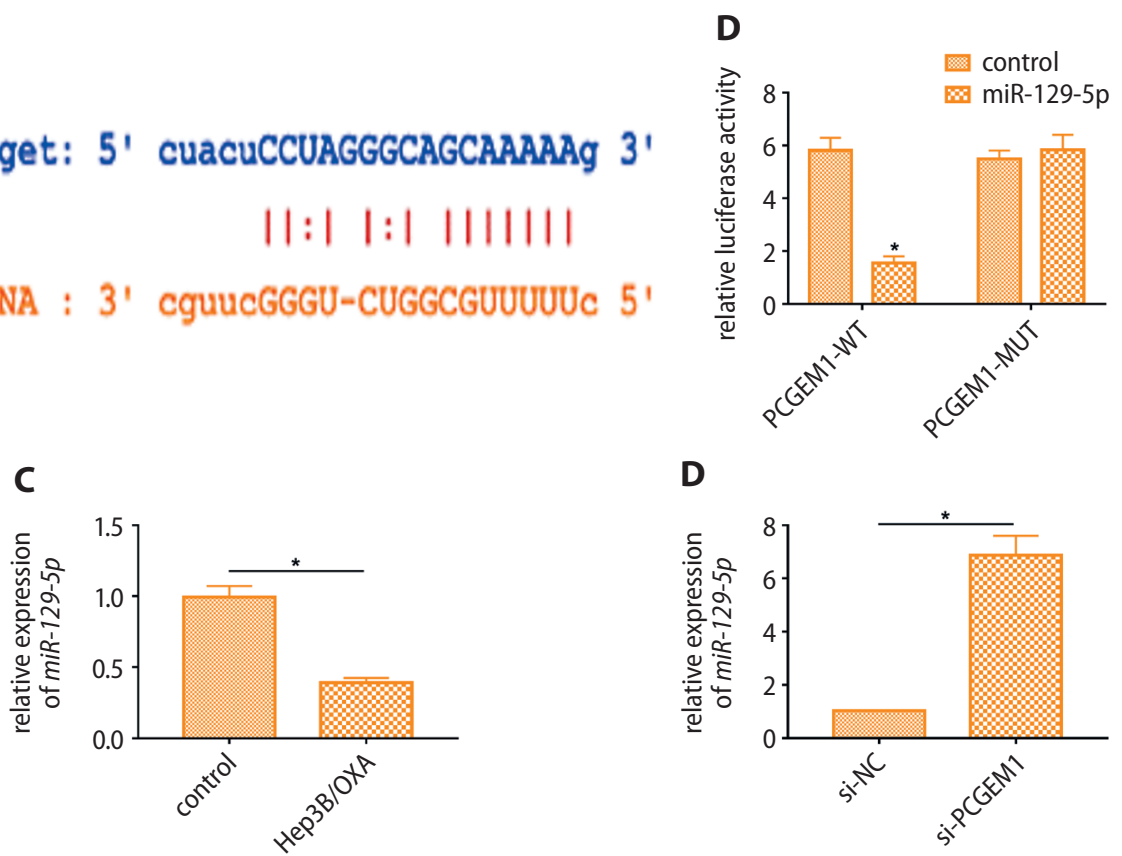

D

prepared and Hep3B/OXA cells were transfected with them to reduce the expression of PCGEM1 (Fig. 1D). Moreover, our findings revealed that PCGEM1 knockdown considerably decreased the mRNA expression of drug resistance-related genes, including low-density lipoprotein receptor-related protein 1 (LRP1), multidrug resistance gene 1 (MDR1) and multidrug resistance gene 3 (MDR3) compared to that of the control group (Fig. 1E). The CCK-8 assay was used to measure the cell growth inhibition curve by calculating the value of the inhibitory concentration $\left(\mathrm{IC}_{50}\right)$. Our findings elucidated that PCGEM1 knockdown markedly suppressed the $\mathrm{IC}_{50}$ value in comparison to the control group (Fig. 1F). These data illustrated that PCGEM1 knockdown markedly reduced the oxaliplatin resistance of HCC cells.

\section{Effect of IncRNA PCGEM1 on invasion and migration in the oxaliplatin-resistant HCC cells}

Transwell assays indicated that PCGEM1 knockdown considerably reduced the number of invasive cells in Hep3B/OXA cells compared to that of the empty vector group (Fig. 2A). Our findings also revealed that PCGEM1 knockdown markedly reduced the number of migrating cells compared to the empty vector cells (Fig. 2B). These findings revealed that PCGEM1 knockdown inhibited the invasion and migration in Hep3B/OXA cells.

\section{MiR-129-5p was a target of PCGEM1}

Online bioinformatics tools including miRcode (http:// mircode.org/), starBase v. 2.0 (http://starbase.sysu.edu.cn/ starbase2/index.php) and microRNA.org (https://bigd.big. ac.cn/databasecommons/database/id/1426), together with a luciferase assay, were used for the prediction and confirmation of binding between PCGEM1 and miR-129-5p. Our data elucidated that the luciferase activity was significantly reduced in the PCGEM1-WT and miR-129-5p mimics group. At the same time, no significant change was observed in the rest of the groups (Fig. 3A,B). Moreover, $m i R-129-5 p$ expression was reduced in Hep3B/OXA cells compared to that of parental Hep3B cells (Fig. 3C). Finally, miR-129-5p expression was significantly enhanced in Hep3B/OXA cells transfected with si-PCGEM1 compared to that of the empty vector group (Fig. 3D). 
A

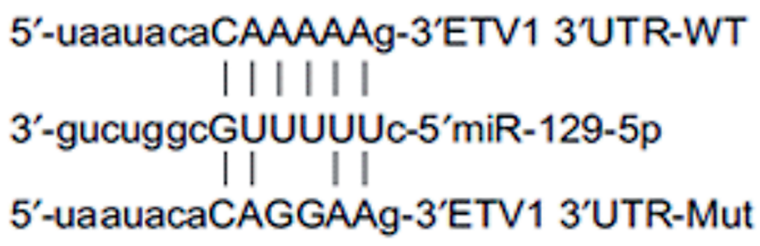

C

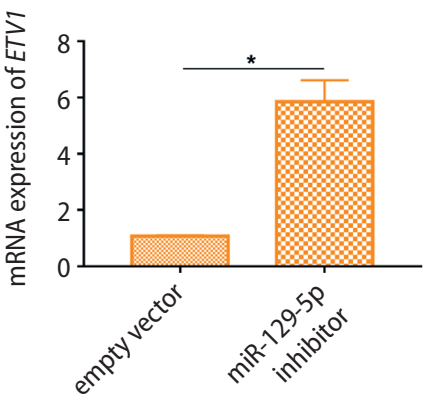

\section{LncRNA PCGEM1 regulated ETV1 expression via targeting miR-129-5p}

The results from bioinformatic tools indicated that miR-129-5p shared binding sites with the 3'UTR of ETV1 mRNA (Fig. 4A). Luciferase assay results revealed a decrease in luciferase activity after the co-transfection with miR-129-5p and ETV1-WT (100 ng), indicating molecular binding between miR-129-5p and ETV1 (Fig. 4B). Furthermore, ETV1 mRNA expression was increased in Hep3B/OXA cells transfected with miR-129-5p inhibitor $(50 \mathrm{nmol} / \mathrm{L})$ (Fig. 4C). Finally, the knockdown of PCGEM1 reduced $E T V 1$ mRNA expression, while inhibition of $m i R$ 129-5p enhanced the mRNA level of ETV1 in Hep3B/OXA cells (Fig. 4D).

\section{Discussion}

The HCC is a hepatic malignant neoplasm. ${ }^{24}$ Despite great advancement in the therapeutic techniques for HCC, the overall outcomes remain unsatisfactory, ${ }^{25}$ with a common obstacle being chemoresistance towards drugs such as oxaliplatin, doxorubicin and cisplatin, among others. ${ }^{26}$ Our study explored the regulatory functions of PCGEM1 in oxaliplatin resistance regarding $m i R-129-5 p / E T V 1$ interactions using oxaliplatin-resistant HCC cells (Hep3B/ OXA) (Fig. 5).

The lncRNAs present a vital function in regulating tumorigenesis, as well as the chemoresistance of cancer cells. ${ }^{27}$ Previous research has suggested that dysregulated
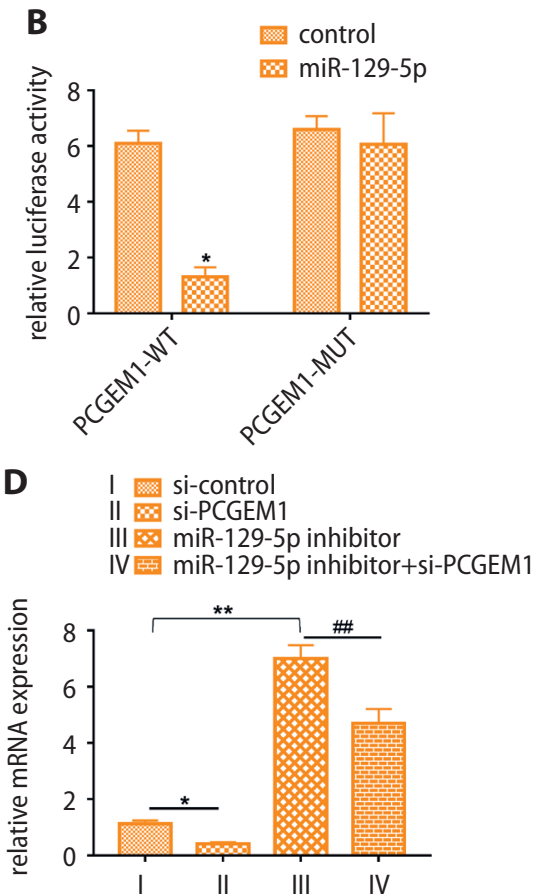

Fig. 4. LnCRNA PCGEM1 regulated ETV1 expression by targeting miR-129-5p. $A$ and $B$. The binding sites between ETV1 and miR-129-5p were predicted using online bioinformatic tools including TargetScan website (http://www.targetscan.org) and starBase website (http://starbase.sysu.edu.cn/ starbase2/index.php), and confirmed using a luciferase assay ( $\left.{ }^{*} \mathrm{p}<0.03\right)$; C. The ETV1 mRNA level was assessed in Hep3B/OXA cells transfected with miR-129-5p inhibitor using qRT-PCR $\left({ }^{*} p<0.03\right.$, compared to si-control); D. The ETV1 mRNA level was measured in Hep3B/OXA cells transfected with an empty vector, miR-129-5p inhibitor and PCGEM1 siRNA ( ${ }^{*} p<0.03$ **p $<0.002$ compared to si-control, $\#$ p $<0.002$ compared to miR-129-5p inhibitor)

lncRNA expression might mediate potential chemoresistance. ${ }^{28}$ Our findings revealed that the PCGEM1 expression was enhanced in Hep3B/OXA cells compared to that of its parental cell line. Furthermore, knockdown of lncRNA PCGEM1 decreased the mRNA expression levels of $L R P 1, M D R 1$ and $M D R 3$ in Hep3B/OXA cells, indicating that silencing of $P C G E M 1$ might reduce the oxaliplatin resistance. Moreover, PCGEM1 knockdown significantly inhibited the proliferation, migration and invasiveness of Hep3B/OXA cells, which was in accordance with the hypothesis that downregulation of PCGEM1 might lead to the decrease in chemoresistance in vitro. Similar to our findings, it has been reported that downregulation of another lncRNA, lncRNA PVT1 was able to inhibit tumor progression and reduced chemoresistance to cisplatin in colorectal cancer patients. ${ }^{29}$ In HCC, IncRNA HULC was significantly upregulated in HCC cells after treatment with drugs including cisplatin and oxaliplatin, and

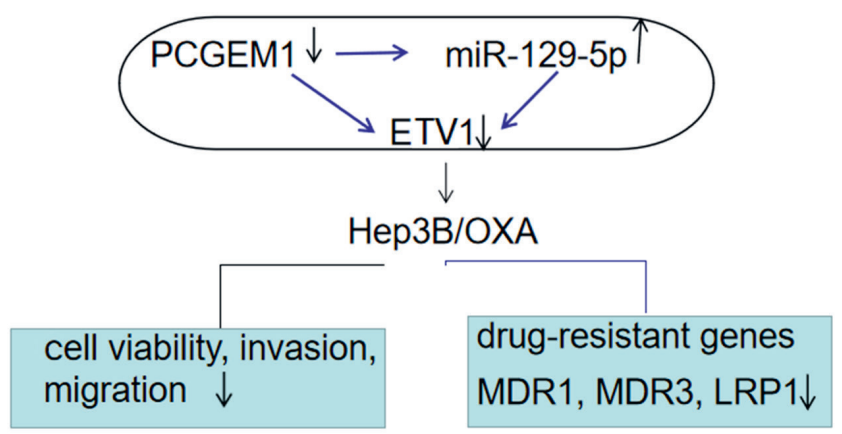

Fig. 5. The illustration of the hypothetical mechanism of PCGEM1 in Hep3B/OXA cells 
the additional knockdown of HULC enhanced chemoresistance of HCC cells through its interaction with $S I R T 1^{19}$. In this study, we found that the relative mRNA expression of ETV1 was decreased by the knockdown of lncRNA PCGEM1 in Hep3B/OXA cells. ETV1 has been reported to function as an oncogene in various human malignant tumors. ${ }^{30,31}$ A previous study indicated that ETV1 enhanced the invasion, migration and proliferation of breast cancer cells. ${ }^{32}$ Moreover, inhibitor of ETV1 enhanced the cellular sensitivity to docetaxel in prostate cancer. ${ }^{33}$ Similarly, ETV1 was reported to be modulated by Circ-ZNF609/ miR-1224-3p in lung cancer cells. ${ }^{34}$ We discovered that the expression of ETV1 was positively regulated by $P C$ GEM1 in Hep3B/OXA cells, suggesting that knockdown of PCGEM1 in HCC cells increased sensitivity to oxaliplatin, perhaps through the modulation of ETV1.

To predict the targeted sites between mRNA and lncRNA, bioinformatic prediction tools were used..$^{35}$ By using these online tools, we established that $m i R-129-5 p$ targeted PCGEM1. The miR-129-5p overexpression has been shown previously to attenuate the proliferation of PC-3 cells, ${ }^{36}$ while in prostate cancer, $m i R-129-5 p$ was downregulated in tissues and cells in comparison with normal counterparts, and it specifically inhibits ETV1. ${ }^{36}$ Furthermore, the upregulation of $m i R-129-5 p$ was shown to curb the cell proliferation by suppressing ETV1 in prostate cancer cells. ${ }^{36}$ Finally, it was disclosed that miR-129-5p inhibited the development of colon cancer and osteosarcoma via regulating $H M G B 1 .{ }^{37,38}$ In conclusion, our data revealed the impact of lncRNA PCGEM1 in HCC oxaliplatin resistance in vitro. We have shown that PCGEM1 and miR-129-5p expression levels were negatively correlated to each other, representing the enrichment and antagonistic function. PCGEM1 downregulation led to an increase in $m i R-129-5 p$ expression in Hep3B/OXA cells, while downregulation of $m i R-129-5 p$ induced ETV1 mRNA expression. In our work, PCGEM1 downregulation reduced the oxaliplatin resistance in $\mathrm{Hep} 3 \mathrm{~B} / \mathrm{OXA}$ cells via binding miR-129-5p and inhibiting ETV1.

\section{Limitations}

The limitation of this current study mainly exists in the absence of animal models. If respective animal models could be established to further validate the mechanism of the PCGEM1/miR-129-5p/ETV1 pathway on HCC oxaliplatin resistance, the research would be more valuable and complete.

\section{Conclusions}

Our findings demonstrated the potential mechanism of the PCGEM1/miR-129-5p/ETV1 pathway on HCC oxaliplatin resistance, suggesting the critical function of $P C$ GEM1 in HCC treatment and providing a new therapeutic target.

\section{ORCID iDs}

Jie Chen (iD https://orcid.org/0000-0003-3904-4564

Daiyue Yuan (D) https://orcid.org/0000-0002-2720-2337

Qingya Hao (D) https://orcid.org/0000-0002-4205-2242

Dongmei Zhu (D) https://orcid.org/0000-0001-7062-8475

Zhong Chen (D) https://orcid.org/0000-0002-1598-9726

\section{References}

1. Brown ZJ, Heinrich B, Steinberg SM, Yu SJ, Greten TF. Safety in treatment of hepatocellular carcinoma with immune checkpoint inhibitors as compared to melanoma and non-small cell lung cancer. J Immunother Cancer. 2017;5(1):93. doi:10.1186/s40425-017-0298-2

2. Li M, Zhang M, Zhang ZL, et al. Induction of apoptosis by berberine in hepatocellular carcinoma HepG2 cells via downregulation of NF-KB. Oncol Res. 2017;25(2):233-239. doi:10.3727/096504016x147 42891049073

3. Dika IE, Abou-Alfa GK. Treatment options after sorafenib failure in patients with hepatocellular carcinoma. Clin Mol Hepatol. 2017;23(4): 273-279. doi:10.3350/cmh.2017.0108

4. Hocquelet A, Aubé C, Rode A, et al. Comparison of no-touch multibipolar vs monopolar radiofrequency ablation for small HCC.J Hepatol. 2017;66(1):67-74. doi:10.1016/j.jhep.2016.07.010

5. Huang X, Lu S. Impact of preoperative locoregional therapy on recurrence and patient survival following liver transplantation for hepatocellular carcinoma: A meta-analysis. Scand J Gastroenterol. 2017;52(2): 143-149. doi:10.1080/00365521.2016.1236396

6. Margonis GA, Sasaki K, Andreatos N, et al. Prognostic impact of complications after resection of early stage hepatocellular carcinoma. J Surg Oncol. 2017;115(7):791-804. doi:10.1002/jso.24576

7. Meng YB, He X, Huang YF, Wu QN, Zhou YC, Hao DJ. Long noncoding RNA CRNDE promotes multiple myeloma cell growth by suppressing miR-451. Oncol Res. 2017;25(7):1207-1214. doi:10.3727/096504017x14 886679715637

8. Qin N, Tong GF, Sun LW, Xu XL. Long noncoding RNA MEG3 suppresses glioma cell proliferation, migration, and invasion by acting as a competing endogenous RNA of miR-19a. Oncol Res. 2017;25(9):1471-1478. doi:10.3727/096504017x14886689179993

9. Liu K, Wu X, Zang $X$, et al. TRAF4 regulates migration, invasion, and epithelial-mesenchymal transition via PI3K/AKT signaling in hepatocellular carcinoma. Oncol Res. 2017;25(8):1329-1340. doi:10.3727/ $096504017 \times 14876227286564$

10. Cao SW, Huang JL, Chen J, et al. Long non-coding RNA UBE2CP3 promotes tumor metastasis by inducing epithelial-mesenchymal transition in hepatocellular carcinoma. Oncotarget. 2017;8(39):65370-65385. doi:10.18632/oncotarget.18524

11. Wang F, Ying HQ, He BS, et al. Upregulated IncRNA-UCA1 contributes to progression of hepatocellular carcinoma through inhibition of miR$216 \mathrm{~b}$ and activation of FGFR1/ERK signaling pathway. Oncotarget. 2015;6(10):7899-7917. doi:10.18632/oncotarget.3219

12. Yang J, Li J, Liu B, et al. Long noncoding RNA AK021443 promotes cell proliferation and migration by regulating epithelial-mesenchymal transition in hepatocellular carcinoma cells. DNA Cell Biol. 2018;37(5): 481-490. doi:10.1089/dna.2017.4030

13. Srikantan V, Zou Z, Petrovics G, et al. PCGEM1, a prostate-specific gene, is overexpressed in prostate cancer. Proc Natl Acad Sci USA. 2000;97(22):12216-12221. doi:10.1073/pnas.97.22.12216

14. Petrovics G, Zhang W, Makarem M, et al. Elevated expression of PCGEM1, a prostate-specific gene with cell growth-promoting function, is associated with high-risk prostate cancer patients. Oncogene. 2004; 23(2):605-611. doi:10.1038/sj.onc.1207069

15. Chen S, Wang LL, Sun KX, et al. LncRNA PCGEM1 induces ovarian carcinoma tumorigenesis and progression through RhoA pathway. Cell Physiol Biochem. 2018;47(4):1578-1588. doi:10.1159/000490931

16. Zhang J, Jin HY, Wu Y, et al. Hypoxia-induced LncRNA PCGEM1 promotes invasion and metastasis of gastric cancer through regulating SNAI1. Clin TransI Oncol. 2019;21(9):1142-1151. doi:10.1007/s12094-01902035-9

17. Seo SU, Min KJ, Woo SM, Kwon TK. Z-FL-COCHO, a cathepsin S inhibitor, enhances oxaliplatin-mediated apoptosis through the induction of endoplasmic reticulum stress. Exp Mol Med. 2018;50(8):107. doi:10.1038/s12276-018-0138-6 
18. Yin X, Zheng SS, Zhang L, et al. Identification of long noncoding RNA expression profile in oxaliplatin-resistant hepatocellular carcinoma cells. Gene. 2017;596:53-88. doi:10.1016/j.gene.2016.10.008

19. Xiong $\mathrm{H}, \mathrm{Ni} \mathrm{Z}, \mathrm{He} \mathrm{J}$, et al. LncRNA HULC triggers autophagy via stabilizing Sirt1 and attenuates the chemosensitivity of HCC cells. Oncogene. 2017;36(25):3528-3540. doi:10.1038/onc.2016.521

20. Bu Y, Jia QA, Ren ZG, et al. Maintenance of stemness in oxaliplatin-resistant hepatocellular carcinoma is associated with increased autocrine of IGF1. PLoS One. 2014;9(3):e89686. doi:10.1371/journal. pone.0089686

21. Kim MK, Kim WT, Lee HM, et al. Correction: Mapping of a mycoplasma-neutralizing epitope on the mycoplasmal p37 protein. PLoSOne. 2017;12(2):e0172487. doi:10.1371/journal.pone.0172487

22. Ma J, Zeng S, Zhang Y, et al. BMP4 promotes oxaliplatin resistance by an induction of epithelial-mesenchymal transition via MEK1/ERK/ ELK1 signaling in hepatocellular carcinoma. Cancer Lett. 2017;411: 117-129. doi:10.1016/j.canlet.2017.09.041

23. Schmittgen TD, Livak KJ. Analyzing real-time PCR data by the comparative C(T) method. Nat Protoc. 2008;3(6):1101-1108. doi:10.1038/ nprot. 2008.73

24. Lu LC, Chang CJ, Hsu CH. Targeting myeloid-derived suppressor cells in the treatment of hepatocellular carcinoma: Current state and future perspectives. J Hepatocell Carcinoma. 2019;6:71-84. doi:10.2147/jhc. s159693

25. Flynn MJ, Sayed AA, Sharma R, Siddique A, Pinato DJ. Challenges and opportunities in the clinical development of immune checkpoint inhibitors for hepatocellular carcinoma. Hepatology. 2019;69(5): 2258-2270. doi:10.1002/hep.30337

26. Liu L, Li N, Zhang Q, Zhou J, Lin L, He X. Inhibition of ERK1/2 signaling impairs the promoting effects of TGF- $\beta 1$ on hepatocellular carcinoma cell invasion and epithelial-mesenchymal transition. Oncol Res. 2017;25(9):1607-1616. doi:10.3727/096504017x14938093512742

27. Liu Z, Zhang H. LncRNA plasmacytoma variant translocation 1 is an oncogene in bladder urothelial carcinoma. Oncotarget. 2017;8(38): 64273-64282. doi:10.18632/oncotarget.19604
28. Chen G, Guo Z, Liu M, et al. Clinical value of capecitabine-based combination adjuvant chemotherapy in early breast cancer: A meta-analysis of randomized controlled trials. Oncol Res. 2017;25(9):1567-1578. doi:10.3727/096504017x14897173032733

29. Ping G, Xiong W, Zhang L, Li Y, Zhang Y, Zhao Y. Silencing long noncoding RNA PVT1 inhibits tumorigenesis and cisplatin resistance of colorectal cancer. Am J Transl Res. 2018;10(1):138-149.

30. Oh S, Shin S, Janknecht R. ETV1, 4 and 5: An oncogenic subfamily of ETS transcription factors. Biochim Biophys Acta. 2012;1826(1):1-12. doi:10.1016/j.bbcan.2012.02.002

31. Chi $P$, Chen Y, Zhang L, et al. ETV1 is a lineage survival factor that cooperates with KIT in gastrointestinal stromal tumours. Nature. 2010;467(7317):849-853. doi:10.1038/nature09409

32. Ouyang $\mathrm{M}$, Wang $\mathrm{H}, \mathrm{Ma}$ J, et al. COP1, the negative regulator of ETV1, influences prognosis in triple-negative breast cancer. BMC Cancer. 2015;15:132. doi:10.1186/s12885-015-1151-y

33. Yu L, Wu X, Chen M, et al. The effects and mechanism of YK-4-279 in combination with docetaxel on prostate cancer. Int J Med Sci. 2017; 14(4):356-366. doi:10.7150/ijms.18382

34. Zuo Y, Shen W, Wang C, Niu N, Pu J. Circular RNA Circ-ZNF609 promotes lung adenocarcinoma proliferation by modulating miR-12243p/ETV1 signaling. Cancer Manag Res. 2020;12:2471-2479. doi:10.2147/ cmar.s232260

35. Zhang G, Yang P. Bioinformatics genes and pathway analysis for chronic neuropathic pain after spinal cord injury. Biomed Res Int. 2017;2017:6423021. doi:10.1155/2017/6423021

36. Gao G, Xiu D, Yang B, et al. miR-129-5p inhibits prostate cancer proliferation via targeting ETV1. Oncotargets Ther. 2019;12:3531-3544. doi:10.2147/ott.s183435

37. Liu K, Huang J, Ni J, et al. MALAT1 promotes osteosarcoma development by regulation of HMGB1 via miR-142-3p and miR-129-5p. Cell Cycle. 2017;16(6):578-587. doi:10.1080/15384101.2017.1288324

38. Wu Q, Meng WY, Jie Y, Zhao H. LncRNA MALAT1 induces colon cancer development by regulating miR-129-5p/HMGB1 axis. J Cell Physiol. 2018;233(9):6750-6757. doi:10.1002/jcp.26383 